\title{
Valence band structure of polytypic zinc-blende/wurtzite GaAs nanowires probed by polarization-dependent photoluminescence
}

\author{
D. Spirkoska, ${ }^{1}$ Al. L. Efros, ${ }^{2}$ W. R. L. Lambrecht, ${ }^{3}$ T. Cheiwchanchamnangij, ${ }^{3}$ A. Fontcuberta i Morral, ${ }^{4}$ and G. Abstreiter ${ }^{1}$ \\ ${ }^{1}$ Walter Schottky Institut and Physik Department, Technische Universität München, Am Coulombwall 4, D-85748 Garching, Germany \\ ${ }^{2}$ Naval Research Laboratory, Washington DC, 20375, USA \\ ${ }^{3}$ Department of Physics, Case Western Reserve University, 10900 Euclid Avenue, Cleveland, Ohio 44106-7079, USA \\ ${ }^{4}$ Laboratoire des Matériaux Semiconducteurs, Institut des Matériaux, Ecole Polytechnique Fédérale de Lausanne, Lausanne, Switzerland
}

(Received 9 October 2011; revised manuscript received 15 December 2011; published 10 January 2012)

\begin{abstract}
We conducted temperature-dependent measurements of the photoluminescence (PL) polarization on GaAs nanowires (NWs) with polytypic zinc-blende/wurtzite structure in order to probe the symmetry and energy structure of the valence band in the wurtzite segments of the NWs. The low-temperature measurements revealed that in most of the investigated cases, the ground level of the interface excitons responsible for the PL is formed by the heavy hole. To describe the observed temperature dependence of the degree of PL polarization, we developed a theoretical model that allows an estimation of the splitting between the heavy hole and light hole exciton subbands in these NWs. This splitting is smaller than expected in pure wurtzite on the basis of recent first-principles calculations, which may be attributed to the multiple twinned nature of the wurtzite sections, which effectively behave as a polytype of lower hexagonality.
\end{abstract}

DOI: 10.1103/PhysRevB.85.045309

PACS number(s): 62.23.Hj, 63.22.Gh, 81.15.Hi, 81.16.Dn

\section{INTRODUCTION}

The field of semiconductor nanowires has noted a tremendous development over the past decade. This is due to the vast application of nanowires (NWs) in a variety of research fields: material science, basic physical phenomena in low-dimensional systems, as well as applied nanowire devices. ${ }^{1-4}$ One of the main advantages of these systems with a high surface to volume ratio is the possibility for studying phenomena not accessible in bulk materials. An astonishing example is the existence of the wurtzite crystal structure in arsenide and phosphide III-V's as well as some II-VI based NWs. Unlike the bulk counterparts, the structure of the NWs can consist of pure phases of zinc-blende or wurtzite, or a mixture of the two phases. ${ }^{5-12}$ Three different aspects of the wurtzite crystal structure in materials that in bulk exist only in zinc-blende form have been studied up to date. First, nucleation theories have been developed to investigate the appearance of wurtzite structure as a direction for obtaining pure crystal phases. ${ }^{13-16}$ Second, the calculations of the energy parameters of the band structure of wurtzite GaAs and $\mathrm{InP}^{17-20}$ have attracted a significant interest. Finally, the energy structure of the zinc-blende wurtzite heterojunction ${ }^{8,9}$ and the piece-wise wurtzite phase material in the $\mathrm{NWs}^{10,21,22}$ have been studied experimentally using photoluminescence (PL) spectroscopy.

From a crystallographic point of view, the difference between the zinc-blende and the wurtzite structure lies in the stacking of the atomic planes in the (111) growth direction. The zinc-blende structure exhibits an ABCABC... stacking sequence, while the wurtzite structure is characterized by the ABABA... stacking of the layers. This is illustrated in Fig. 1(a), which shows schematically the stacking of the different layers along the NW growth direction. The change of the layer stacking that occurs on the scale larger than several nanometers leads to alternation of the zinc-blende and wurtzite structures and formation of multiple zinc-blende/wurtzite heterointerfaces.
The exact values of the band gaps of the wurtzite phase materials are still under discussion. For example, several values for the band gap of the wurtzite GaAs have been calculated. $^{18,20,23}$ The theoretically calculated values differ from each other due to their sensitivity on the used computational methods and the applied structural parameters of relaxed GaAs. The most recent theoretical calculations ${ }^{20}$ agree well with the measured value by resonant Raman spectroscopy. ${ }^{24}$ On the other hand, theories agree that the zinc-blende/wurtzite heterointerface exhibits a staggered-type II band alignment, as schematically presented in Fig. 1(b). ${ }^{17,18,25}$ The electrons are confined in the zinc-blende segments, while the holes are localized in the wurtzite segments. The weak overlap of the wave functions of the spatially separated electrons and holes results in a long decay time of the PL, which was confirmed by time-resolved measurements. ${ }^{8,26}$ Only recently, however, the symmetry and energy structure of the valence band of such zinc-blende/wurtzite polytypic structures was measured experimentally in InP and heterostructured $\mathrm{GaAs} / \mathrm{GaAsSb}$ NWs using polarization-dependent PL and (or) PL excitation spectroscopy. $5,21,22,27$

In this paper, we study the symmetry and energy structure of the exciton bands in GaAs NWs with zinc-blende structure as well as polytypic GaAs NWs with mixed zinc-blende/wurtzite structure by measuring the PL polarization properties at different temperatures. In the first case, the PL is connected with recombination of weakly confined excitons in zinc-blende GaAs NWs, while in the later case, the PL is represented by multiple lines connected with an indirect electron-hole pair recombination at the zinc-blende/wurtzite heterointerfaces. At low temperature, the emission of the weakly confined excitons in zinc-blende GaAs NWs is polarized parallel to the NW growth direction, while most of the PL lines studied in various zinc-blende/wurtzite NWs are polarized perpendicular to the NW growth direction indicating the heavy hole exciton to be the ground energy level. Temperature elevation decreases their polarization ratio and eventually can even reverse the 
(a)

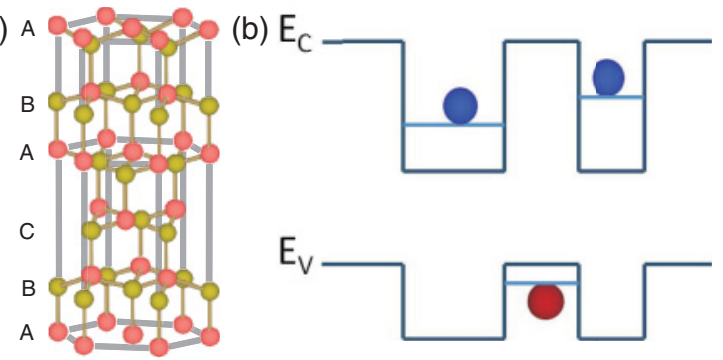

FIG. 1. (Color online) (a) Illustration of the modifications of the NW crystal structure along the growth direction. The zincblende structure exhibits $\mathrm{ABCABC}$...stacking of the layers along the (111) growth direction, while the wurtzite structure is characterized by $\mathrm{ABAB}$...stacking sequence. (b) Band alignment at the zincblende/wurtzite heterointerface. The crystal phases exhibit type II-staggered band alignment with spatially separated electron and hole. The electron is localized in the zinc-blende segment, while the hole is found in the wurtzite segment. Succession of the zinc-blende and wurtzite segments creates multiple heterointerfaces perpendicular to the nanowire.

PL polarization. The decrease and reversal are interpreted as a result of thermal population of an excited state with a dipole parallel to the NW growth axis. This state couples much stronger with light than the ground state due to the absence of dielectric screening of the light electric field vector parallel to the NW growth axis. A theoretical model that describes the PL polarization temperature dependence was developed. Analysis of the experimental data on the polarization dependence in terms of a nanowire confinement model allowed us to extract the effective splitting of the heavy and light hole bands in the corresponding bulk crystal. However this splitting is found to be smaller than the value in wurtzite. This is explained in terms of the multiple twinned nature of the nanowires, which leads to a splitting intermediate between zincblende and wurtzite.

The paper is organized as follows: In Sec. II, we present the detailed description of our experimental setup. The experimental results and their preliminary analysis will be given in Sec. III. Section IV is providing a theoretical description of the energy spectra and polarization properties of excitons weakly confined in GaAs NWs. The analyses of the experimental data using the developed theory and the discussion of the obtained results will be conducted in Sec. V.

\section{EXPERIMENTAL DETAILS}

We have grown GaAs NWs by applying the Ga-assisted molecular beam epitaxy (MBE) growth method. These nanowires have typical diameters of $80-100 \mathrm{~nm}$ and a length of 5-6 $\mu \mathrm{m}$. Details of the NWs' growth can be found elsewhere. ${ }^{28-30}$ In our previous work, we have investigated the different growth regimes under which the crystal structure of the NWs can be changed from pure zinc-blende to a mixture of zinc-blende and wurtzite with different wurtzite content. ${ }^{8}$ The experiments revealed that NWs grown under high growth rate exhibit mostly zinc-blende structure with the occasional presence of twin defects, while NWs grown under medium and low growth rate have polytypic zinc-blende/wurtzite structure with increased content of wurtzite. In the present work, we have concentrated on NWs exhibiting in an average $70 \%$ of zinc-blende and $30 \%$ of wurtzite structure.

The nanowires consist of regions with pure almost twin free zinc-blende segments but also regions with alternating zinc-blende and wurtzite segments and highly twinned zincblende structure. The wurtzite segments have typical lengths of a few monolayers up to a few tens of nanometers. The highly twinned zinc-blende part of the structure is characterized with very small intertwin spacing (a few nanometers in average). High resolution transmission electron microscopy (HR TEM) micrograph from a part of one nanowire is shown in Fig. 2(a). Here, rotational twin defects in the zinc-blende structure (labeled with green and blue circles) but also segments with wurtzite structure (labeled with red circles) can be identified. A detailed TEM analysis of the appearance of segments with different crystal structure along the nanowire growth direction can be found in Ref. 8. In accordance with the model of zinc-blende/wurtzite heterojunction, quantum wells are formed along the growth direction of the NWs, emitting at different energies due to the different confined energy levels.

To conduct PL measurements on a single NW, the NWs were removed from the growth substrate and transferred subsequently on oxidized Si pieces by applying a mechanical

(a)
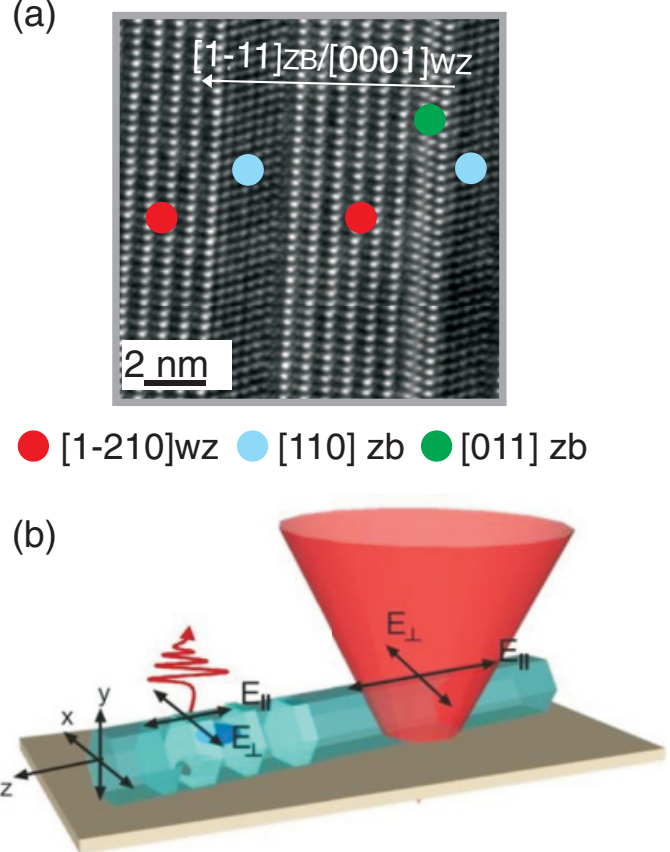

FIG. 2. (Color online) (a) High resolution TEM micrograph from a short portion of a GaAs nanowire. The structure consists of zinc-blende with rotational twin defects (green and blue circles) and wurtzite (red circles) segments. The nanowire growth direction is also indicated: [1-11] for the zinc-blende segments and [0001] for the wurtzite segments. A detailed TEM analysis of the appearance of the different segments along the nanowire growth direction can be found in Ref. 8. (b) Schematic presentation of the experimental configuration for polarization-dependent PL measurements. The NW growth direction is along the $z$ axis. The polarization of the incident laser light can be changed from parallel to perpendicular with respect to the NW growth direction. The polarization of the emitted PL is analyzed by a second polarizer. 
contact. The measurements were performed in a confocal microscopy configuration with a base temperature of $4.2 \mathrm{~K}$. The sample was mounted on a stage with four piezo positioners. Three of them allow movement in the $x, y$, and $z$ direction, while the fourth is a fine scanner for movement in the $x y$ plane. We used a laser diode emitting at $785 \mathrm{~nm}$ (photon energy $E=1.579 \mathrm{eV}$ ) as an excitation source. An objective lens $(\mathrm{NA}=0.65)$ focused the laser light on to the sample with a spot diameter of about 600 to $700 \mathrm{~nm}$. The same objective lens is used to collect the emitted PL, which was further analyzed with a combination of a grating spectrometer with a focal distance of the mirror $f=30 \mathrm{~cm}$ and $d=1200$ grooves $/ \mathrm{mm}$ on the grating and a Peltier oooled CCD. A resistive heater mounted in close vicinity to the sample allows a controllable increase of the temperature. By performing reflectivity measurements, while scanning the sample under the laser spot, single NWs can be localized. For polarization-dependent PL measurements, additional optical elements were mounted in the paths of incident as well as emitted light. A set of lambda-half plates and a linear polarizer allowed a controllable change of the polarization of the incident laser light from parallel to perpendicular to the nanowire axis. An additional linear polarizer was used for analysis of the polarization of the PL lines. The experimental configuration is illustrated in Fig. 2(b).

\section{EXPERIMENTAL RESULTS}

We have investigated the polarization properties of the PL emission from many individual nanowires exhibiting pure zinc-blende structure with occasional twin defects as well as intermittent zinc-blende/wurtzite structure (total amount of wurtzite is about $30 \%$ ). In the following, we will first elaborate the polarization properties of nanowires with zinc-blende structure and then continue with considering the nanowires with intermittent zinc-blende/wurtzite structure.

\section{A. Nanowires with zinc-blende structure}

A typical spatially resolved PL spectrum from a single nanowire is presented in Fig. 3(a). The PL from the zinc-blende structure nanowires is characterized by a single emission peak, centered at $1.515 \mathrm{eV}$, corresponding to the free exciton energy of bulk GaAs. The PL characterization clearly suggests that the nanowires are of very high quality as no or only very weak defect related luminescence is observed. Furthermore, the nanowire emits along the entire length. A PL spectrum recorded at the position of the dashed line is presented in Fig. 3(b). The PL is characterized by a single peak centered at $1.515 \mathrm{eV}$. The full width at half maximum (FWHM) is around $5 \mathrm{meV}$.

Next, we measured the intensity of the emitted PL with respect to the polarization of the incident laser beam. The emitted PL was integrated over all polarizations. The angular dependence of the integrated PL intensity on the polarization of the incident laser is plotted in Fig. 3(c). For clarity, an image of the NW acquired by reflectivity measurements is superimposed. The circles are the experimental data points,

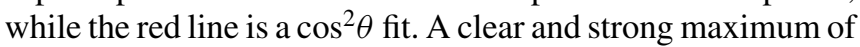
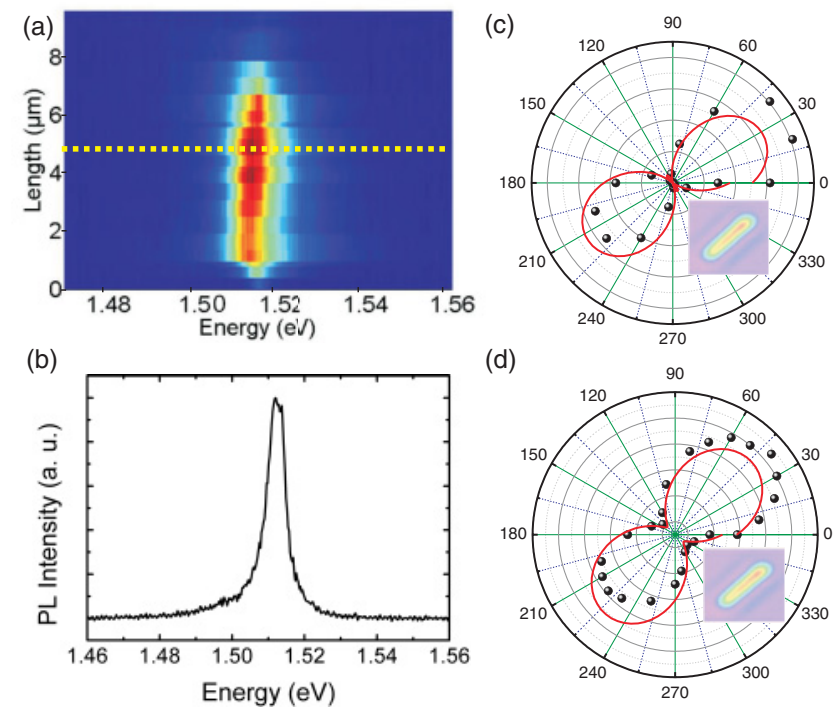

FIG. 3. (Color online) (a) Spatially resolved PL from a single NW with zinc-blende structure. The PL emission extends along the entire length. (b) PL spectrum recorded at the position of the dashed line in (a). (c) Angular dependence of the integrated PL intensity on the polarization of the excitation laser. For clarity, an image of the NW is also superimposed. (d) Polarization properties of the emitted PL. The incident laser is polarized along the NW growth direction. An image of the NW is also superimposed.

PL intensity is obtained when the excitation laser is polarized along the NW growth direction.

Figure 3(d) shows the polarization properties of the ground exciton state in the zinc-blende GaAs NW. The PL intensity was recorded for several different angles between the linear polarizer and the NW growth direction. The corresponding polar plot is presented in Fig. 3(d), where an image of the NW is also superimposed. Clearly, the emitted PL is also polarized along the NW growth direction. In order to maximize the absorption, the polarization of the excitation laser was chosen parallel to the NW growth direction, although similar polarization dependence was obtained for different polarization of exciting light. This shows that the observed PL polarization is an intrinsic property of the ground exciton state in the NW, and it is not connected with PL polarization memory effect. In the plots presented in Figs. 3(c) and 3(d), it can be noticed that the $\cos ^{2} \theta$ fit of the experimental data points is not a closed function. This results from losses in the PL signal during the measurements influenced by small misalignment of the experimental setup as the angle of the polarizers is changed. This is also causing the small inconsistency (approximately $10^{\circ}$ ) between the maximum of the PL in Figs. 3(c) and 3(d) with respect to the nanowire growth direction.

The highly polarized absorption and emission from zincblende GaAs NWs has already been observed in the past ${ }^{1}$ and has been assigned to the large dielectric mismatch between the nanowire and the environment. ${ }^{31}$ The degree of PL polarization defined as $P=\left(I_{\|}-I_{\perp}\right) /\left(I_{\|}+I_{\perp}\right)\left(I_{\|}\right.$and $I_{\perp}$ are the intensities of emitted light polarized parallel and perpendicular to the NW growth direction) in these NWs is as high as $92 \pm 2 \%$. 


\section{B. Nanowires with intermittent zinc-blende/wurtzite structure}

The PL spectra from the NWs with intermittent zincblende/wurtzite crystal structure exhibit, besides the emission at $1.515 \mathrm{eV}$, several other emission lines at lower energies connected with recombination of indirect excitons at the multiple zinc-blende/wurtzite hetero-interfaces formed along the NW growth direction, as discussed in detail in Ref. 8. A typical example is presented in Fig. 4(a), where the spatially resolved PL spectrum is shown. Indeed, the PL emission of the NW is characterized by several emission lines with different energies originating from the different parts of the NW. All these lines have energies smaller than the free exciton emission of zinc-blende GaAs $(E=1.515 \mathrm{eV})$ marked by the white arrow, which is only observed in one part of the NW. In Ref. 8, we have presented a detailed study of these low energy emission lines including time resolved studies, demonstrating their spatially indirect nature. A PL spectrum recorded at the position marked with the yellow dashed line is presented in Fig. 4(b). The total PL spectrum is a convolution of several different emission peaks. We have fitted the entire spectrum with multiple Lorentzian lines. All measurements presented later in this paper are performed at this position of the NW.

To study the PL polarization properties of the spatially separated electron-hole pairs, we excite the NWs with the laser, which polarization was chosen parallel to the NW axis. The PL intensity was recorded for different angles $\theta$ between the NW growth direction and the linear polarizer. For each $\theta$, the recorded PL spectrum was fitted using multiple Lorentzian lines that allow us to extract the relative integral intensities of the different emission energies. The polarization properties of several PL lines from the spectrum presented in Figs. 4(a) and 4(b) are plotted in Fig. 4(c). For clarity, an image of the NW is also shown. It is obvious that the emission lines are polarized perpendicular to the NW growth direction. The degree of PL polarization lies in the range between 78 and $98 \%$.

We have found that more than $90 \%$ of these low-lying energy lines in all investigated NWs are polarized perpendicular to the NW axis at low temperature, in strong contrast with the emission originating from the free exciton in zinc-blende GaAs NWs (polarized along the NW growth direction as discussed above). Similar results have been published recently on GaAs nanowires with predominant wurtzite structure but with the presence of thin zinc-blende segments. ${ }^{27}$ In the following, we present a detailed temperature study of the polarization behavior of selected emission lines from the spectrum presented in Fig. 4(a). Figures 5(a) and 5(b) show the temperature modifications of the dependence of the PL intensity on the polarization angle $\theta$, for two emission lines with energies $E=1.476 \mathrm{eV}$ and $E=1.483 \mathrm{eV}$. We have chosen these two lines because we can clearly resolve them up to a temperature of $14 \mathrm{~K}$. This reduces the error in determining the integral intensity of the peaks. The experimental data points are shown by symbols. The polarization dependence is well

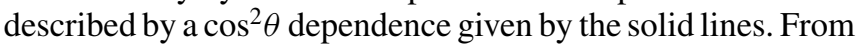
the polar plots, it is evident that the maximum PL emission intensity is obtained for polarization perpendicular to the NW growth direction for the entire temperature range, however the degree of polarization decreases with increasing temperature. For temperatures larger then $14 \mathrm{~K}$, the PL intensity of these
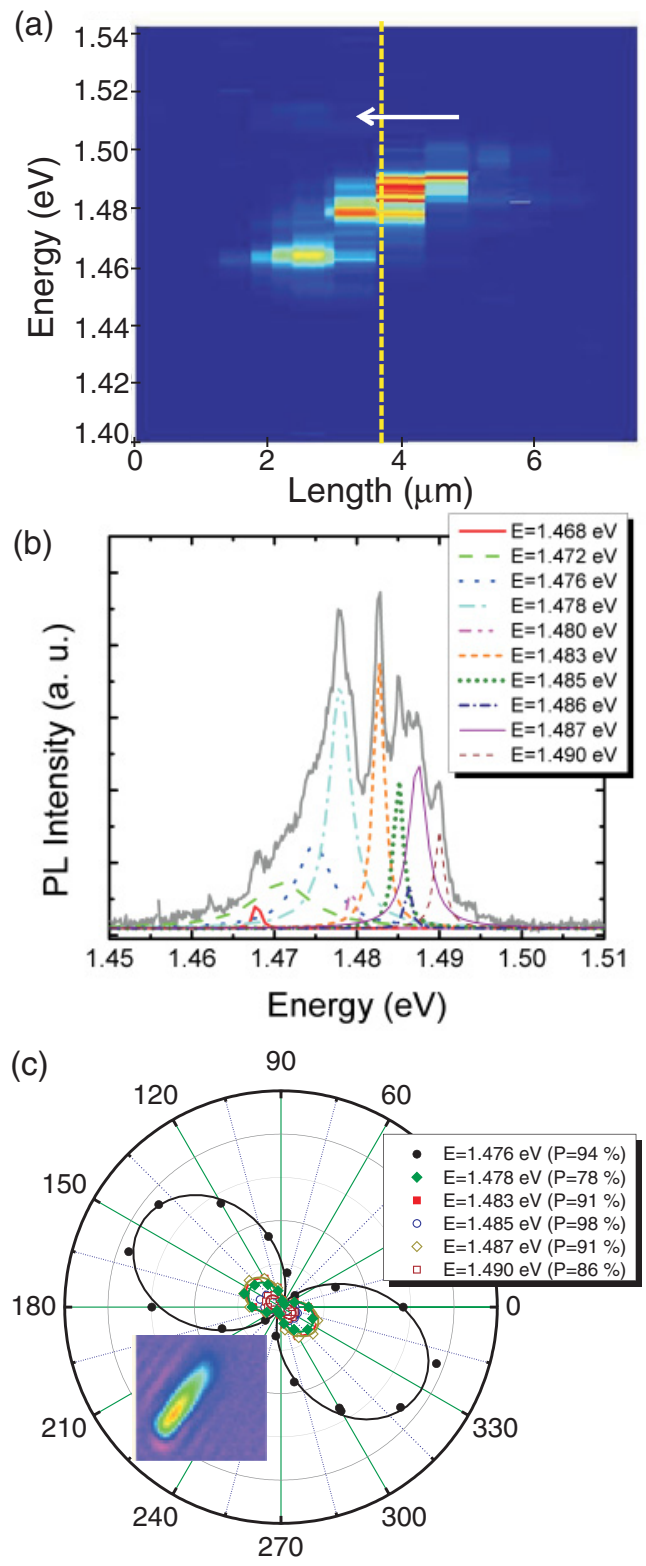

FIG. 4. (Color online) (a) Spatially resolved PL spectrum from a single GaAs NW exhibiting mixture of zinc-blende and wurtzite crystal structure with different thicknesses of the crystal phase segments. The PL is characterized with several emission energies in the range between $1.450 \mathrm{eV}$ and $1.515 \mathrm{eV}$, which sources are localized at different segments along the NW growth direction. The free exciton emission energy in zinc-blende GaAs is shown by the white arrow (b). PL spectrum was recorded at the position of the dashed line in (a). The resulting multiline spectrum is characterized by a convolution of several peaks with different emission energies shown in the inset. (c) Polarization dependence of several PL lines from the spectrum in (b). Clearly, the emissions are polarized perpendicular to the NW growth direction (an image of the nanowire is superimposed).

lines is strongly reduced due to the small exciton binding energy.

As discussed above and demonstrated in detail in Ref. 8, the studied low-energy PL lines are due to recombination of electron-hole pairs spatially separated at the 
(a)

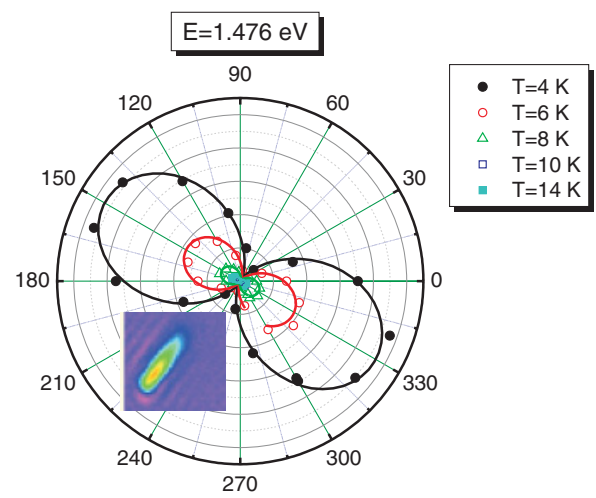

(b)

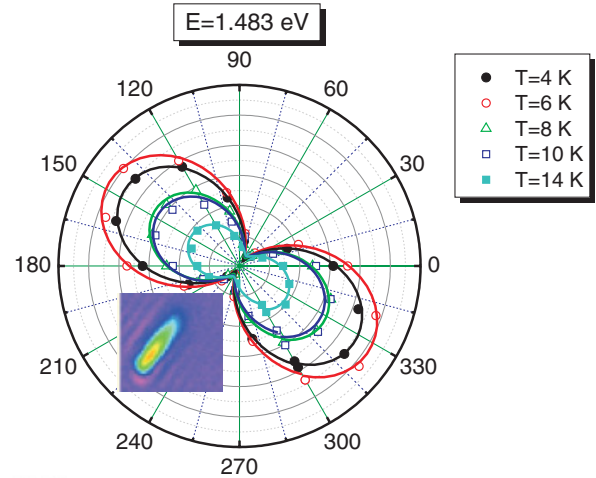

(c)

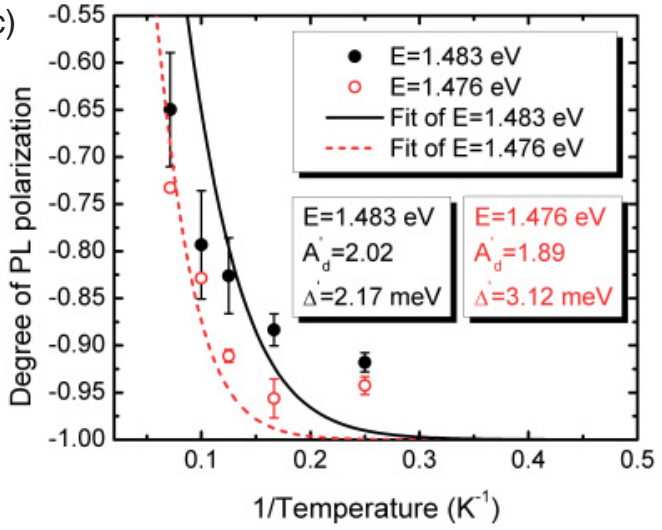

FIG. 5. (Color online) (a),(b) Dependence of the PL intensity on the angle between the NW growth direction and the linear polarizer for the emission energies $1.476 \mathrm{eV}$ and $1.483 \mathrm{eV}$ recorded at several different temperatures. An image of the NW required in scanning reflection mode is superimposed on the polar plots. (c) Temperature dependence of the degree of PL polarization for the two different emission energies in (a) and (b). The symbols are presenting the experimental data. The lines show a fit of these data using Eq. (6) with $A_{d}^{\prime}$ and $\Delta^{\prime}$ as the fitting parameters.

zinc-blende/wurtzite interface, with the electron localized in the zinc-blende part and the hole in the wurtzite part. The Bloch function of the conduction band in the zinc-blende GaAs has $S$-like symmetry. As a consequence, the polarization properties of the emitted light are determined by the symmetry of the Bloch function of holes in the valence band of the wurtzite GaAs. The top of the valence band in wurtzite GaAs is split in three levels by a combination of spin-orbit and crystal field splittings. The levels labeled by the double group notation are

$$
\begin{aligned}
\Gamma_{9} & =\Delta_{c}+\Delta_{1 s}, \\
\Gamma_{7 \pm} & =\frac{\Delta_{c}-\Delta_{s 1}}{2} \pm \sqrt{\left(\frac{\Delta_{c}-\Delta_{s 1}}{2}\right)^{2}+2 \Delta_{s 2}^{2}} .
\end{aligned}
$$

Here, $\Delta_{c}$ is the crystal field splitting and $\Delta_{1 s}, \Delta_{2 s}$ are two spin-orbit coupling parameters both about $1 / 3$ of the cubic spin-orbit splitting. In $\mathrm{GaAs}$, the spin-orbit coupling is large compared to the crystal field splitting, so the lower $\Gamma_{7-}$ state can be ignored and the upper heavy hole $\left(\Gamma_{9}\right)$ and light hole $\left(\Gamma_{7+}\right)$ states are split in energy by a splitting which we denote simply as $\Delta .{ }^{32}$ The polarization of the interband optical transitions is controlled by the symmetry of the corresponding Bloch functions, which can be written as ${ }^{33}$

$$
\begin{aligned}
& u_{ \pm 3 / 2}=\frac{1}{\sqrt{2}}(X \pm i Y) \uparrow, \\
& u_{ \pm 1 / 2}=\frac{i}{\sqrt{6}}[(X \pm i Y) \downarrow \mp 2 Z \uparrow],
\end{aligned}
$$

where $\mu$ is the projection of the total angular momentum on the $\mathrm{NW}$ growth direction and equals to $\mu= \pm 1 / 2$ and $\mu= \pm 3 / 2$ for the light hole and heavy hole states, respectively. One can see that at the top of the valence band, the heavy holes have dipoles only along the $x$ and $y$ directions, perpendicular to the NW growth $z$ direction, and only the excited light hole states have a nonzero dipole along $z$.

The PL polarization properties are controlled by the matrix element squared $\left|\left\langle S|\hat{\boldsymbol{p}} \cdot \boldsymbol{E}| u_{\mu}\right\rangle\right|^{2}$, where $|S\rangle$ is the Bloch function of the conduction band electron that has $s$-like spherical symmetry in the zinc-blende structure. Therefore, we need to find an electric field of an emitted photon of certain polarization $\boldsymbol{E}$ within the NW and various algebraic combinations of the matrix elements $\left\langle S\left|\hat{p}_{x}\right| X\right\rangle=\mathcal{P},\left\langle S\left|\hat{p}_{y}\right| Y\right\rangle=\mathcal{P}$ and $\left\langle S\left|\hat{p}_{z}\right| Z\right\rangle=\mathcal{P}$. Each matrix element equals to the Kane matrix element $\mathcal{P}$. The expressions for the Bloch function in Eq. (2) show that both the light and heavy holes could contribute to the emission of light, which is polarized perpendicular to the $\mathrm{NW}$ growth direction. The corresponding intensities of the PL can be written as

$$
I_{\perp}^{h} \sim\left(\frac{\left\langle S\left|\hat{p}_{x}\right| X\right\rangle}{\sqrt{2}}\right)^{2}=\frac{\mathcal{P}^{2}}{2}, I_{\perp}^{l} \sim\left(\frac{\left\langle S\left|\hat{p}_{x}\right| X\right\rangle}{\sqrt{6}}\right)^{2}=\frac{\mathcal{P}^{2}}{6}
$$

for the heavy hole and light hole transitions, respectively. One can see from Eq. (3), that the heavy holes contribute three times more efficiently than the light holes to the light polarized perpendicular to the NW.

On the other hand, one can see from Eq. (2) that only the light holes can contribute light polarized parallel to the NW growth direction ( $z$ direction). Furthermore, the PL polarized along the NW growth direction is enhanced relative to the one polarized perpendicular to the NW growth direction due to the dielectric confinement effect. ${ }^{31}$ The dielectric confinement factor $A_{d}$ in NWs can be written as

$$
A_{d}=\left(\frac{\epsilon+\epsilon_{0}}{2 \epsilon_{0}}\right)^{2},
$$

where $\epsilon$ and $\epsilon_{0}$ are the dielectric constant of semiconductor and surrounding medium, respectively. As a result, the PL intensity 
of the light polarized parallel to the NW growth direction is proportional to

$$
I_{\|}^{l} \sim A_{d}\left(\frac{2\left\langle S\left|\hat{p}_{z}\right| Z\right\rangle}{\sqrt{6}}\right)^{2}=A_{d} \frac{2 \mathcal{P}^{2}}{3} .
$$

For the case of GaAs nanowire in vacuum $(\epsilon=10.8$ and $\left.\epsilon_{0}=1\right), A_{d}=35.2$. To describe the degree of the PL polarization, we assume that the excitons are completely thermalized before their radiative recombination. The relative population of the exciton level, which has light hole character, to the population of the exciton level, which has heavy hole character, is described by the Boltzmann factor $\exp \left(-\Delta / k_{B} T\right)$, where $\Delta$ is the energy separation between light hole and heavy hole states, $k_{B}$ is the Boltzmann constant, and $T$ is the temperature. If the ground exciton level has heavy hole character, then $\Delta$ is positive, and it is negative if the ground state has a light hole character. The above consideration shows that the PL polarized parallel to the NW growth direction could only be a result of light hole exciton recombination. This polarization is always enhanced by the dielectric confinement factor $A_{d}$, but requires the thermal population of the levels of the light hole exciton.

The high degree of PL polarization observed in zinc-blende GaAs NWs at liquid helium temperature (92\%) is a clear indication that the lowest exciton level in these structures is connected with the light hole. The maximum theoretical value of the PL polarization degree, $\left(4 A_{d}-1\right) /\left(4 A_{d}+1\right) \approx 98 \%$, could be reached if only the light hole exciton is populated. At temperatures larger than the level splitting, when both the light and heavy hole excitons are equally populated, the polarization degree is described as $\left(A_{d}-1\right) /\left(A_{d}+1\right) \approx 92 \%$. The deviation from the maximum value and the weak temperature dependence of the PL polarization degree connected with the population of the heavy hole exciton was measured previously in InP NWs. ${ }^{1}$

Photoluminescence polarized perpendicular to the NW growth direction observed in the zinc-blende/wurtzite NWs clearly indicates that the ground exciton state is connected with heavy holes. In the wurtzite segments of the NWs, additional factors could contribute to the total splitting of the heavy and light hole subbands besides the bulk splitting $\Delta$ of wurtzite GaAs: confinement of holes along the NW growth direction and confinement connected with NW finite thickness, as well as various strains measured in these NWs. ${ }^{34}$ We will denote the effective energy difference between light and heavy hole subbands in the nanowires as $\Delta^{\prime}$. Similarly, the nanowire states have no longer pure $\mu= \pm 3 / 2$ or $\mu= \pm 1 / 2$ character. This affects the effective ratio $A_{d}$ and we will thus label it as $A_{d}^{\prime}$ in the nanowire. Taking into account the intensity of the PL emitted parallel and perpendicular to the NW growth direction [see Eqs. (4) and (5)] and thermal populations of the light and heavy hole exciton states, one can write for the PL polarization degree

$$
P=\frac{4 A_{d}^{\prime} \exp \left(-\Delta^{\prime} / k_{B} T\right)-3-\exp \left(-\Delta^{\prime} / k_{B} T\right)}{4 A_{d}^{\prime} \exp \left(-\Delta^{\prime} / k_{B} T\right)+3+\exp \left(-\Delta^{\prime} / k_{B} T\right)} .
$$

From this expression, one can see that the temperature increase, which leads to population of the light hole states, results eventually in a reversal of the PL polarization: from perpendicular to parallel.
Figure 5(c) shows the temperature dependence of the degree of PL polarization $P$ for the two emission lines. The error bars on the experimental data points were extracted from the errors in the Lorentzian fit of the spectrum. The degree of PL polarization, which was around $90 \%$ at the lowest temperature $(4.2 \mathrm{~K})$, clearly decreases with the temperature rise. We relate this behavior to the thermal population of the light hole states. The lines show the fit of the experimental data by the theoretical dependence described by Eq. (6), with $A_{d}^{\prime}$ and $\Delta^{\prime}$ used as fitting parameters. The best fit parameters are shown in the graph legend. For both emission lines, $A_{d}^{\prime}$ was found to be close to 2 , while $\Delta^{\prime}$ varies between $2 \mathrm{meV}$ and $3 \mathrm{meV}$. It was found that there is a considerable variation in these parameters between different nanowires and even in different sections of the same nanowire: $\Delta^{\prime}$ varied from 0.53 to $6.78 \mathrm{meV}$ and $A_{d}^{\prime}$ from 0.1 to 2.02 .

The extracted values differ significantly from the theoretically expected values. As discussed above, the corresponding bulk $A_{d}$ should have a value of 35.2 . In addition, a significantly larger value for the crystal field splitting $\Delta \sim 100 \mathrm{meV}$ is expected in wurtzite GaAs. ${ }^{20}$ In the next sections, we will address these issues in terms of a model that includes the size-quantization effects in the nanowire. The model will also need to account for the variability of these parameters; in other words, some other property is changing in the nanowires.

\section{EXCITONS IN GaAs NANOWIRES}

To describe these unexpected results, we developed a theory for the energy spectra of the zinc-blende/wurtzite interface excitons in GaAs NWs. In the studied NWs, the exciton Bohr radius is much smaller than the NW radius. As a result, the energy spectra of such excitons can be considered in the so-called weak confinement regime, when the radial-transverse to the NW growth axis-confinement is affecting only the exciton center-of mass-motion. ${ }^{35,36} \mathrm{In}$ this case, the two-dimensional motion of the indirect exciton along the interface can be described by the quasi-Luttinger Hamiltonian with effective crystal field splitting term $\Delta$, which lifts the degeneracy of the light and heavy hole excitons at zero momentum. ${ }^{37}$

\section{A. Excitons in zinc-blende GaAs nanowires}

We will start, however, from the description of the exciton spectra in zinc-blende NWs. In the weak confinement limit, the exciton center motion can be described by the effective Luttinger-Kohn Hamiltonian ${ }^{38}$

$$
\hat{H}_{L K}=\left(\gamma_{1}+\frac{5}{2} \gamma\right) \frac{\hat{p}^{2}}{2 m_{0}}-\frac{1}{m_{0}}(\hat{\boldsymbol{p}} \cdot \boldsymbol{J})^{2},
$$

where $\hat{\boldsymbol{p}}$ is the exciton center-of-mass momentum operator, $m_{0}$ is the mass of the free electron, $J_{x, y, z}$ are the spin matrices of the $3 / 2$ momentum, ${ }^{39}$ and $\gamma_{1}$ and $\gamma$ are the Luttinger parameters connected with the effective masses of the light, $M_{l}=m_{0} /\left(\gamma_{1}+2 \gamma\right)$, and heavy, $M_{h}=m_{0} /\left(\gamma_{1}-2 \gamma\right)$, excitons, respectively.

In NWs with cylindrical symmetry, each exciton center-ofmass motion state described by the Luttinger-Kohn Hamiltonian of Eq. (7) is characterized by the total angular momen- 
tum projection $F=m+\mu$, which is a sum of the angular momentum projection $m$ and the spin projection of the hole $\mu= \pm 1 / 2, \pm 3 / 2$. The wave functions of the corresponding states can be generally written in the following form:

$$
\Psi^{F}(\rho, \phi, z)=\sum_{\mu=-3 / 2}^{3 / 2} u_{\mu} \chi_{\mu}^{F}(\rho) \exp [i(F-\mu) \phi] \exp \left(i k_{z} z\right)
$$

where $\rho=\sqrt{x^{2}+y^{2}}$ and $z$ are the coordinates across and along the NW growth axis, $\phi$ is the azimuthal angle, $k_{z}$ is the exciton quasi-momentum along the $z$ axis, and $u_{\mu}$ are the Bloch functions of the degenerate valence bands defined in Eq. (2). The optically active excitons have $k_{z}=0$. Substituting Eq. (8) into the Hamiltonian described by Eq. (7) and putting $k_{z}=0$, we obtain the two independent equations defining the radial functions $\chi_{\mu}^{F}(\rho)$ of the optically active excitons:

$$
\begin{aligned}
& \left(\frac{\hbar^{2}}{2 m_{0}}\left(\gamma_{1}+\gamma\right) \Delta_{F-3 / 2}-E\right) \chi_{3 / 2}^{F}(\rho)+\frac{\sqrt{3} \hbar^{2} \gamma}{2 m_{0}} \hat{D}_{F-1 / 2}^{-} \hat{D}_{F+1 / 2}^{-} \chi_{-1 / 2}^{F}(\rho)=0, \\
& \frac{\sqrt{3} \hbar^{2} \gamma}{2 m_{0}} \hat{D}_{F-1 / 2}^{+} \hat{D}_{F-3 / 2}^{+} \chi_{3 / 2}^{F}(\rho)+\left(\frac{\hbar^{2}}{2 m_{0}}\left(\gamma_{1}-\gamma\right) \Delta_{F+1 / 2}-E\right) \chi_{-1 / 2}^{F}(\rho)=0
\end{aligned}
$$

and

$$
\begin{aligned}
& \left(\frac{\hbar^{2}}{2 m_{0}}\left(\gamma_{1}-\gamma\right) \Delta_{F-1 / 2}-E\right) \chi_{1 / 2}^{F}(\rho)+\frac{\sqrt{3} \hbar^{2} \gamma}{2 m_{0}} \hat{D}_{F+1 / 2}^{-} \hat{D}_{F+3 / 2}^{-} \chi_{-3 / 2}^{F}(\rho)=0, \\
& \frac{\sqrt{3} \hbar^{2} \gamma}{2 m_{0}} \hat{D}_{F+1 / 2}^{+} \hat{D}_{F-1 / 2}^{+} \chi_{1 / 2}^{F}(\rho)+\left(\frac{\hbar^{2}}{2 m_{0}}\left(\gamma_{1}+\gamma\right) \Delta_{F+3 / 2}-E\right) \chi_{-3 / 2}^{F}(\rho)=0,
\end{aligned}
$$

where $E$ is the state energy and the differential operators $\hat{D}_{m}^{ \pm}$

$$
\hat{D}_{m}^{ \pm}=\mp \frac{\partial}{\partial \rho}+\frac{m}{\rho}
$$

are the raising and lowering operators $\hat{D}_{m}^{ \pm} J_{m}(k \rho)=k J_{m \pm 1}(k \rho)$ for the Bessel functions with integer index $J_{m}(k \rho)$ and the operator $\Delta_{m}=\hat{D}_{m+1}^{-} \hat{D}_{m}^{+}=-(1 / \rho)(\partial / \partial \rho) \rho(\partial / \partial \rho)+m^{2} / \rho^{2}$.

The confinement of the exciton motion across the NW axis results in quantization of the exciton energy spectrum. We found the energy spectrum and the corresponding wave function of the exciton center-of-mass motion assuming that the exciton wave function vanishes at the NW surface. Using two independent systems of the differential Eqs. (9) and (10), we obtain two equations that describe the energy spectra of confined excitons in zinc-blende NWs:

$$
3 J_{F-3 / 2}(\eta \beta) J_{F+1 / 2}(\eta)+J_{F+1 / 2}(\eta \beta) J_{F-3 / 2}(\eta)=0,
$$

and

$$
J_{F-1 / 2}(\eta \beta) J_{F+3 / 2}(\eta)+3 J_{F+3 / 2}(\eta \beta) J_{F-1 / 2}(\eta)=0,
$$

where $\beta=\sqrt{M_{l} / M_{h}}$ is the ratio of the light to heavy hole exciton effective mass and $\eta$ 's are the dimensionless roots of Eqs. (12) and (13). The energy of the confined exciton levels $E$, are expressed through $\eta(\beta)$ :

$$
E=\frac{\hbar^{2}}{2 M_{h} a^{2}} \eta^{2}(\beta),
$$

where $a$ is the NW radius.

The optical selection rules and the relative oscillator transition strength of different exciton center-of-mass motion states are determined by the square of the following overlap integral of the hole and electron envelope functions: ${ }^{35} I_{F, n}=$ $\int \rho d \rho d \phi \Psi_{n}^{F}(\rho, \phi)$, where $n$ is counting the exciton states with the given symmetry $n=1,2, \ldots$. The integration over $\phi$ leaves only one term with $\mu=F$ in the integral and reduces it to the following form:

$$
I_{F, n}=2 \pi u_{F} \int \rho d \rho \chi_{F, n}^{F}(\rho),
$$

for $|F| \leqslant 3 / 2$. Excitons with larger $|F|$ are optically passive. One can see from Eq. (15) that excitons with $F= \pm 3 / 2$ possess polarization properties of the heavy holes, while the $F= \pm 1 / 2$ excitons should behave as light holes.

The ground heavy hole exciton state has total angular momentum projections $F= \pm 3 / 2$, and its energy can be found substituting $F=3 / 2$ in Eq. (12):

$$
3 J_{0}\left(\eta^{h} \beta\right) J_{2}\left(\eta^{h}\right)+J_{2}\left(\eta^{h} \beta\right) J_{0}\left(\eta^{h}\right)=0 .
$$

The ground light hole exciton state has total angular momentum projections $F= \pm 1 / 2$, and its energy can be found substituting $F=1 / 2$ in Eq. (13):

$$
J_{0}\left(\eta^{l} \beta\right) J_{2}\left(\eta^{l}\right)+3 J_{2}\left(\eta^{l} \beta\right) J_{0}\left(\eta^{l}\right)=0
$$

The numerical solutions of Eqs. (16) and (17) are presented in Fig. 6 and show that light hole exciton is the ground level for all ratios of light to heavy hole exciton masses $\beta$. The order of the optically allowed transition and the PL polarization properties in zinc-blende NWs were studied previously only in the strong confinement regime. ${ }^{40}$ The calculations demonstrated that the highest confinement level of the valence band in the NWs is connected with the light hole.

The splitting between the light and heavy hole exciton in our NWs with radius in the range of $50 \mathrm{~nm}$ can be found from 


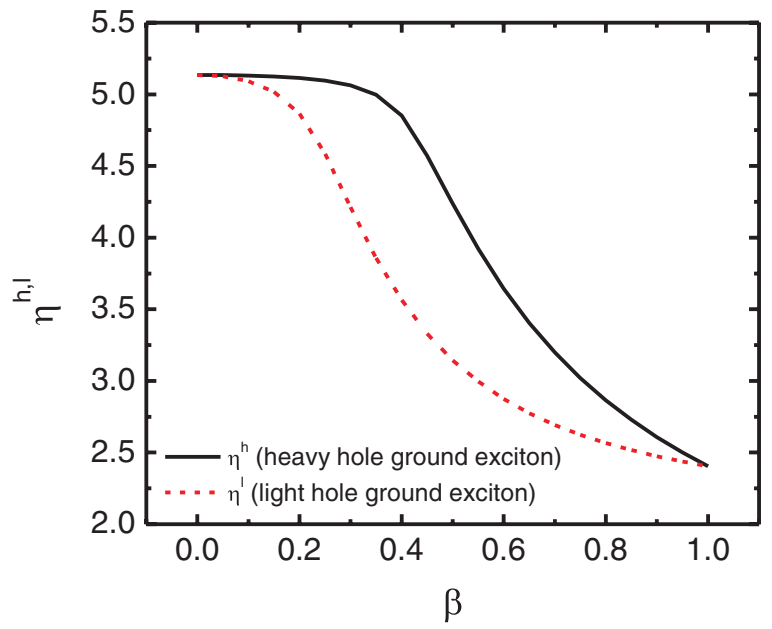

FIG. 6. (Color online) The dependence of the numerical solutions of Eqs. (16) and (17), $\eta^{h}$, and Eq. (17), $\eta^{l}$, for the ground state excitons on the ratio of the light to heavy hole exciton effective mass $\beta$.

Eq. (14) and gives us a value of around $1 \mathrm{meV}$. Using this splitting for the degree of PL polarization [using Eq. (6) with a temperature of $4.2 \mathrm{~K}$ ], we calculated a value of $93 \%$, which is in very good agreement with our experimental data.

\section{B. Interface excitons in zinc-blende/wurtzite GaAs nanowires}

The two-dimensional motion of spatially indirect excitons that are created at the interface between zinc-blende and wurtzite pieces of GaAs NWs could be described by the Hamiltonian that is used for the description of two-dimensional holes in wurtzite semiconductors. The effective Hamiltonian of the exciton motion can be derived using the quasicubic approximation. ${ }^{33}$ In this approximation, the Hamiltonian that describes holes in wurtzite semiconductors is derived by adding to the cubic Hamiltonian $\hat{H}_{L K}$, from Eq. (7), a term $\Delta\left(5 / 4-J_{z}^{2}\right) / 2$, where $\Delta$ is the splitting between $\Gamma_{9}$ and the upper $\Gamma_{7}$ states, as defined in Eq. (1). This adds $\pm \Delta / 2$ to the $\pm 3 / 2, \pm 1 / 2$ states respectively. The approximation is valid as long as the $\Gamma_{7-}$ state lies significantly lower than the $\Gamma_{7+}$ state, so that we need to deal only with the upper four states.

Using this approximation, we derived the Hamiltonian $\hat{H}_{I E}$ that describes two-dimensional motion of the interface excitons

$$
\hat{H}_{I E}=\left.\hat{H}_{L K}\right|_{p_{z}=0}+\Delta\left(5 / 4-J_{z}^{2}\right) / 2,
$$

where $\Delta$ is the total splitting of the light and heavy hole exciton branches at zero transverse momenta $p_{x}=p_{y}=0$. From Eq. (18), one can obtain the momentum $p_{\perp}=\sqrt{p_{x}^{2}+p_{y}^{2}}-$ dispersion of the two branches of the interface excitons:

$$
E_{ \pm}=\gamma_{1} E_{p} \pm \sqrt{4 \gamma^{2} E_{p}^{2}-\gamma E_{p} \Delta+(\Delta / 2)^{2}},
$$

where $E_{p}=p_{\perp}^{2} / 2 m_{0}$. The analysis of the exciton dispersion shows that one cannot separate the heavy excitons from the light ones as soon as the exciton momentum $p_{\perp} \neq 0$, and vice versa. As a result, the heavy hole exciton wave function gains the component of the light hole Bloch function if $p_{\perp} \neq 0$.

In NWs with cylindrical symmetry, each interface exciton state described by Eq. (18) is characterized by the total angular momentum projection $F$, and its wave function can be generally written as

$$
\Psi^{F}(\rho, \phi)=\sum_{\mu=-3 / 2}^{3 / 2} u_{\mu} \chi_{\mu}^{F}(\rho) \exp [i(F-\mu) \phi],
$$

similar to the wave function of excitons in cylindrical zincblende NWs in Eq. (8) with $k_{z}=0$. Now, the radial functions $\chi_{\mu}^{F}(\rho)$ satisfy the system of radial Eqs. (9) and (10) with terms $\frac{\Delta}{2}\left(5 / 4-\mu^{2}\right) \chi_{\mu}^{F}(\rho)$ added to their diagonal elements. The confinement of the exciton motion across the NW axis results in quantization of the exciton energy spectrum. We found the energy spectrum and the corresponding wave function of the exciton center-of-mass motion assuming the exciton wave function vanishes at the NW surface. ${ }^{37}$

(a)
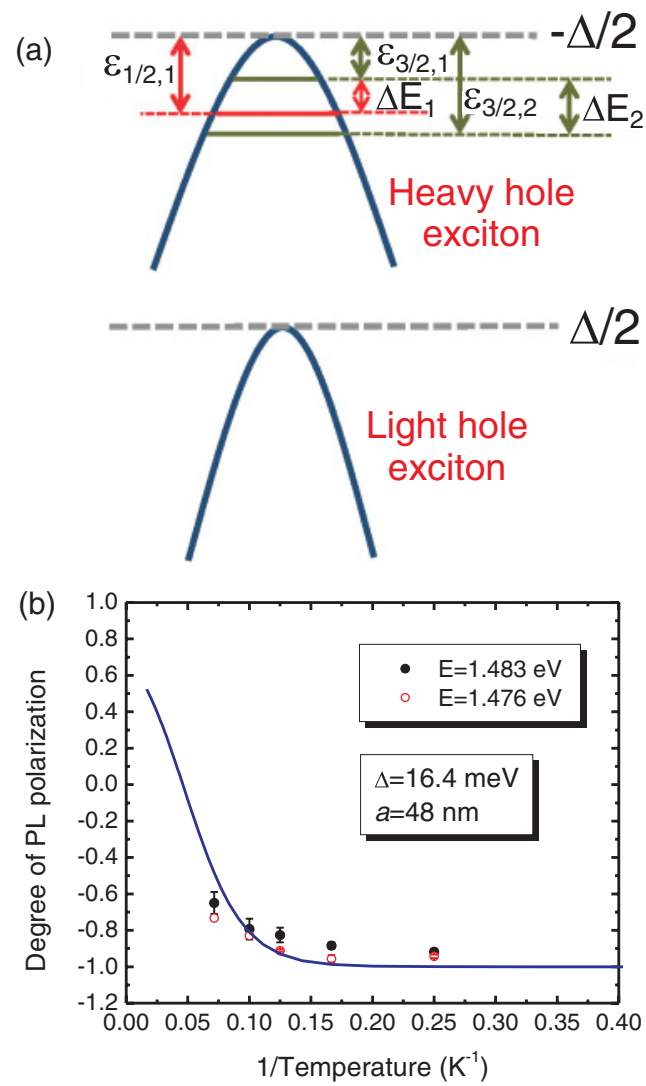

FIG. 7. (Color online) (a) Schematic illustration of the dispersion and the lowest levels of the excitons created at wurtzite/zinc-blende interfaces of intermittent GaAs NWs. $\Delta$ is the energy separation between the heavy hole and light hole exciton subbands. The three lowest optically active exciton levels are shown. The two states with energies $\varepsilon_{3 / 2,1}$ and $\varepsilon_{3 / 2,2}$ have polarization properties of heavy holes, and the third state with energy $\varepsilon_{1 / 2,1}$ has the polarization property of a light hole. $\Delta E_{1}$ and $\Delta E_{2}$ are the energy difference between two excited states and the ground exciton state. (b) The dependence of the degree of PL polarization on temperature for the two emissions (filled and open circles) is fitted in the frames of the three exciton model. The energy $\Delta$ and the NW radius $a$ are used as fitting parameters. The best description gives us $\Delta=16.4 \mathrm{meV}$ and $a=48 \mathrm{~nm}$. 
A full presentation of the wurtzite equation and its numerical solution will be presented elsewhere. ${ }^{37}$ Here, however, we will discuss only the results of the calculation, ${ }^{37}$ which can be obtained assuming that the exciton confined energy $\varepsilon_{F, n}$ is much smaller than the splitting between light and heavy hole excitons $\Delta$, which is always correct in sufficiently thick NWs studied in our experiments. The calculation shows that the ground exciton is a two-fold degenerate state with $|F|=3 / 2$, the second level is a state with $|F|=1 / 2$, and the third level is again a state with $|F|=3 / 2$. The energy of these levels can be written as $E_{F, n}=-\Delta / 2+\varepsilon_{F, n}$, where $n$ is counting the roots of the equations that describe the exciton states with different angular momentum projection $F$, the generalizations of Eqs. (12) and (13) for wurtzite NW. For the lowest optically active state, the energy of these levels can be written as

$\varepsilon_{3 / 2,1} \approx \frac{(2.4 \hbar)^{2}}{2 m_{c} a^{2}}, \quad \varepsilon_{1 / 2,1} \approx \frac{(5.1 \hbar)^{2}}{2 m_{c} a^{2}}, \quad \varepsilon_{3 / 2,2} \approx \frac{(5.5 \hbar)^{2}}{2 m_{c} a^{2}}$

where $m_{c}=m_{0} /\left(\gamma_{1}+\gamma\right)$ is the effective mass of the exciton center-of-mass motion. The structure of the lowest exciton levels is shown schematically in Fig. 7(a). The band diagram shows the two lowest heavy hole exciton levels $\varepsilon_{3 / 2,1}$ and $\varepsilon_{3 / 2,2}$, as well as the light hole exciton level $\varepsilon_{1 / 2,1}$. The last one appears below $\Delta$ as a result of the mixing between the light and heavy hole exciton subbands.

Taking into account the thermal population of the exciton states, the polarization properties, and the values of the corresponding overlap integrals $I_{\mu, n}$ described in Eq. (15), one can write the degree of PL polarization connected with the recombination of the interface excitons. In order to compare it with our previous analysis of the experimental data, we write it initially assuming that there are just two exciton levels separated by the energy $\Delta E_{1}=\varepsilon_{1 / 2,1}-\varepsilon_{3 / 2,1}$. In this two-level approximation,

$$
P=\frac{4 e^{-\Delta E_{1} / k T} I_{1 / 2,1}^{2} A_{d}-I_{1 / 2,1}^{2} e^{-\Delta E_{1} / k T}-3 I_{3 / 2,1}^{2}}{4 e^{-\Delta E_{1} / k T} I_{1 / 2,1}^{2} A_{d}+I_{1 / 2,1}^{2} e^{-\Delta E_{1} / k T}+3 I_{3 / 2,1}^{2}} .
$$

Comparison of Eq. (22) with Eq. (6), which we used to fit the experimental data, shows clearly that the parameter $\Delta^{\prime}$ extracted from the fitting is the distance between the two lowest exciton levels $\Delta E_{1}$, and its small value is determined by the large thickness of the NWs. The other fitting parameter $A_{d}^{\prime}$ in turn can be rewritten as $A_{d}^{\prime}=\left(I_{1 / 2,1}^{2} / I_{3 / 2,1}^{2}\right) A_{d}$, explaining the small experimental value of $A_{d}^{\prime}$. Indeed, $I_{1 / 2,1}$ is proportional to a small admixture of the light hole exciton wave function into the heavy hole exciton wave function connected with the exciton motion perpendicular to the NW growth axis. Under the condition that $\varepsilon_{1 / 2,1} \ll \Delta, A_{d}^{\prime} \propto\left(\varepsilon_{1 / 2,1} / \Delta\right)^{2} A_{d}$ and the first factor significantly reduces the fitting parameter $A_{d}^{\prime}$.

\section{ANALYSES OF EXPERIMENTAL DATA AND DISCUSSION}

From the experimental data in Fig. 5(c), it is evident that even though the degree of PL polarization is decreasing as the temperature is increasing, its reversing to the parallel polarization occurs significantly slower than one could expect. The reason is the small energy difference between the exciton level with light hole character and the second exciton level with heavy hole character. As the temperature is increased, the second exciton level with heavy hole character is populated, keeping the initial orientation of the PL polarization. Therefore, to explain the experimental temperature dependence of PL polarization, we take the third exciton level into account.

The degree of PL polarization in the three level approximation is described by

$$
P=\frac{4 A_{d} I_{1 / 2,1}^{2} e^{-\Delta E_{1} / k T}-3 I_{3 / 2,1}^{2}-I_{1 / 2,1}^{2} e^{-\Delta E_{1} / k T}-3 I_{3 / 2,2}^{2} e^{-\Delta E_{2} / k T}}{4 A_{d} I_{1 / 2,1}^{2} e^{-\Delta E_{1} / k T}+3 I_{3 / 2,1}^{2}+I_{1 / 2,1}^{2} e^{-\Delta E_{1} / k T}+3 I_{3 / 2,2}^{2} e^{-\Delta E_{2} / k T}},
$$

where $\Delta E_{2}=\varepsilon_{3 / 2,2}-\varepsilon_{3 / 2,1}$. We fitted the experimental temperature dependence of the degree of PL polarization by the last expression with the energy splitting $\Delta$ and the NW radius $a$ used as fitting parameters. The best fit for these two specific PL emission lines, shown by the blue line in Fig. 7(b), was reached for $\Delta=16.4 \mathrm{meV}$ and $a=48 \mathrm{~nm}$. The extracted value for the NW radius $a$ is in good accordance with the measured diameter of the nanowires by transmission as well as scanning electron microscopy. ${ }^{8}$

The value extracted here for $\Delta$, the effective $\Gamma_{9}-\Gamma_{7+}$ splitting is significantly smaller than the value of about $100 \mathrm{meV}$ recently calculated by Cheiwchanchamnangij and Lambrecht ${ }^{20}$ for wurtzite GaAs. The latter was found to be close to the value $(103 \mathrm{meV})$ extracted from recent resonant Raman spectroscopy. ${ }^{24}$ The crystal field splitting parameter of GaAs, $\Delta_{c}$, which is the splitting between the $\Gamma_{5}$ and $\Gamma_{1}$ levels in the absence of spin-orbit splitting, was found in Ref. 20 to be sensitive to strain. A uniaxial compression could reduce the value, but estimates based on the strain deformation potentials given in that paper show that a compressive strain of at least $1.3 \%$ is required to obtain values of order $16 \mathrm{meV}$ for $\Delta$. This appears unlikely. Note that $\Delta=\Delta_{c} / 2+3 \Delta_{s 1} / 2-$ $\sqrt{\left(\Delta_{c}-\Delta_{s 1}\right)^{2} / 4+2 \Delta_{s 2}^{2}}$, where $\Delta_{s 1}$ and $\Delta_{s 2}$ are the two spin-orbit coupling parameters in wurtzite, which are 115 and $113 \mathrm{meV}$.

Recently, another possibility was pointed out by Cheiwchanchamnangij et al.: ${ }^{41}$ Polytypes of lower hexagonality such as $4 \mathrm{H}-\mathrm{GaAs}$ have smaller crystal field splitting. Such polytypes, which correspond to alternative stackings, are commonly seen in $\mathrm{SiC}$ but have in fact recently been observed in nanowire GaAs. ${ }^{42}$ One can assign a degree of hexagonality to the polytype as the number of layers that are hexagonally stacked over the total number of layers. ${ }^{43-45}$ For example, $2 \mathrm{H}$ is $100 \%, 4 \mathrm{H}$ is $50 \%, 6 \mathrm{H}$ is $33 \%$ hexagonal and so on. In fact, these authors found that the 
crystal field splitting depends nonlinearly on the degree of hexagonality $h$ :

$$
\Delta_{c}(h)=h \Delta_{c}(1)+b h(1-h),
$$

introducing a "bowing" parameter $b=-84 \mathrm{meV}$ and $\Delta_{c}(1)=$ $180 \mathrm{meV}$. For example, in $4 \mathrm{H}-\mathrm{GaAs}$, which is $50 \%$ hexagonal, $\Delta_{c}=69 \mathrm{meV}$ and $\Delta$ would be $45 \mathrm{meV}$. For hexagonality $h \approx 0.20$ (i.e., a $10 \mathrm{H}$ polytype), $\Delta$ would become of the order of $16 \mathrm{meV}$ as extracted from the experimental data.

Even if regular polytypes do not occur, it seems plausible that if random but very closely spaced stacking faults occur in GaAs, effectively switching the stacking from hexagonal to cubic every few layers, one could assign an effective hexagonality to such multiply twinned regions. This could then significantly reduce the parameter $\Delta$ to the values we deduced, to be compatible with the observed polarization degree of the PL. Slight compressive strain along the nanowires could further reduce $\Delta$, but it is presently unclear what would be the origin of the strain. Since the PL beam averages over a significant region of the NW in which multiple twinning is commonly observed in the electron microscopy images, we think that this effective lower hexagonality is the main reason for our low values of $\Delta$. In other words, the wurtzite NW are not purely wurtzite but effectively behave like a polytype of lower hexagonality. This would furthermore explain why there could be some variation from sample to sample in the NW in their degree of polarization depending on their degree of hexagonality. Further study will be required to fully understand the systematics of these variations and their correlation with the degree of twinning observed in the nanowires.

\section{SUMMARY}

In summary, we have studied the polarization-dependent PL of zinc-blende GaAs NWs and polytypic GaAs NWs, connected with radiative decay of spatially indirect excitons created at zinc-blende/wurtzite interfaces. We found that the PL polarization in zinc-blende GaAs NWs is always parallel to the NW growth direction. This is not the case, however, for polytypic GaAs NWs where the PL polarization is perpendicular to the NW growth direction, although its value decreases significantly with increase of temperature. We have developed a theoretical model that explains these temperature dependences considering the polarization properties of the lowest confinement levels of the exciton center of mass motion across the NW growth axis. From the analysis of our experimental data, we extracted $16 \mathrm{meV}$ splitting between the heavy and light hole exciton subbands in the studied NWs. This value is lower than that in pure wurtzite. Recent first principles calculations suggest that this can be explained by the multiply twinned nature of the wurtzite sections of the NW, which effectively lower their degree of hexagonality.

\section{ACKNOWLEDGMENTS}

The authors thank Prof. J. Arbiol and Prof. J. Morante for the TEM analysis on the nanowires as well as the TEM facilities in the Serveis Cientificotécnics in Universitat de Barcelona. This research was supported by the fundings provided by the Marie Curie Excellence Grant SENFED, the DFG excellence initiative Nanosystems Initiative Munich, and the SFB 631, as well as the Institute of Advanced Study of the Technische Universität München. A. L. E. acknowledges support of the Office of Naval Research and the Alexandervon-Humboldt Foundation.
${ }^{1}$ J. Wang, M. S. Gudiksen, X. Duan, Y. Cui, and C. M. Lieber, Science 293, 1455 (2011).

${ }^{2}$ L. J. Lauhon, M. S. Gudiksen, D. Wang, and C. M. Lieber, Nature (London) 420, 57 (2002).

${ }^{3}$ B. Tian, X. Zheng, T. J. Kempa, Y. Fang, N. Yu, G. Yu, J. Huang, and C. M. Lieber, Nature (London) 449, 885 (2007).

${ }^{4}$ F. A. Zwanenburg, C. E. W. M. van Rijmenam, Y. Fang, C. M. Lieber, and L. P. Kouwenhoven, Nano Lett. 9, 1071 (2009).

${ }^{5}$ A. Mishra, L. V. Titova, T. B. Hoang, H. E. Jackson, L. M. Smith, J. M. Yarrison-Rice, Y. Kim, H. J. Joyce, Q. Gao, H. H. Tan, and C. Jagadish, Appl. Phys. Lett. 91, 263104 (2007).

${ }^{6}$ R. E. Algra, M. A. Verheijen, M. T. Borgström, L. -F. Feiner, G. Immink, W. J. P. van Enckevort, E. Vlieg, and E. P. A. M. Bakkers, Nature (London) 456, 369 (2008).

${ }^{7}$ P. Caroff, K. A. Dick, J. Johansson, M. E. Messing, K. Deppert, and L. Samuelson, Nature Nanotechnology 4, 50 (2009).

${ }^{8}$ D. Spirkoska, J. Arbiol, A. Gustaffson, S. Conesa-Boj, F. Glas, I. Zardo, M. Heigoldt, M. H. Gass, A. L. Bleloch, S. Estrade, M. Kaniber, J. Rossler, F. Peiro, J. R. Morante, G. Abstreiter, L. Samuelson, and A. Fontcuberta i Morral, Phys. Rev. B 80, 245325 (2009).

${ }^{9}$ N. Akopian, G. Patriarche, L. Liu, J. -C. Harmand, and V. Zwiller, Nano Lett. 10, 1198 (2010).
${ }^{10}$ T. B. Hoang, A. F. Moses, H. L. Zhou, D. L. Dheeraj, B. O. Fimland, and H. Weman, Appl. Phys. Lett. 94, 133105 (2009).

${ }^{11}$ J. Bao, D. C. Bell, F. Capasso, J. B. Wagner, T. Mårtensson, J. Trägårdh, and L. Samuelson, Nano Lett. 8, 836 (2008).

${ }^{12}$ Z. Wu, Y. Zhang, J. Zheng, X. Lin, X. Chen, B. Huang, H. Wang, K. Huang, S. Li, and J. Kang, J. Mater. Chem. 21, 6020 (2011).

${ }^{13}$ T. Akiyama, K. Sano, N. Kohji, and T. Ito, Jpn. J. Appl. Phys. 45, L275 (2006).

${ }^{14}$ F. Glas, J. -C. Harmand, and G. Patriarche, Phys. Rev. Lett. 99, 146101 (2007)

${ }^{15}$ V. G. Dubrovskii and N. V. Sibirev, Phys. Rev. B 77, 035414 (2008).

${ }^{16}$ P. Krogstrup, S. Curiotto, E. Johnson, M. Aagesen, J. Nygård, and D. Chatain, Phys. Rev. Lett. 106, 125505 (2011).

${ }^{17}$ M. Murayama and T. Nakayama, Phys. Rev. B 49, 4710 (1994).

${ }^{18}$ A. De and C. E. Pryor, Phys. Rev. B 81, 155210 (2010).

${ }^{19}$ M. Heiss, S. Conesa-Boj, J. Ren, H. H. Tseng, A. Gali, A. Rudolph, E. Uccelli, F. Peiro, J. R. Morante, D. Schuh, E. Reiger, E. Kaxiras, J. Arbiol, and A. Fontcuberta i Morral, Phys. Rev. B 83, 045303 (2011).

${ }^{20}$ T. Cheiwchanchamnangij and W. R. L. Lambrecht, Phys. Rev. B 84, 035203 (2011). 
${ }^{21}$ S. Perera, K. Pemasiri, M. A. Fickenscher, H. E. Jackson, L. M. Smith, J. Yarrison-Rice, S. Paiman, Q. Gao, H. H. Tan, and C. Jagadish, Appl. Phys. Lett. 97, 023106 (2010).

${ }^{22}$ E. G. Gadret, G. O. Dias, L. C. O. Dacal, M. M. de Lima Jr., C. V. R. S. Ruffo, F. Iikawa, M. J. S. P. Brasil, T. Chiaramonte, M. A. Cotta, L. H. G. Tizei, D. Ugarte, and A. Cantarero, Phys. Rev. B 82, 125327 (2010).

${ }^{23}$ Z. Zanolli, F. Fuchs, J. Furthmüller, U. von Barth, and F. Bechstedt, Phys. Rev. B 75, 245121 (2007).

${ }^{24}$ B. Ketterer, M. Heiss, E. Uccelli, J. Arbiol, and A. Fontcuberta i Morral, ACS Nano 5, 7585 (2011).

${ }^{25}$ J. L. Birman, Phys. Rev. 115, 1493 (1959).

${ }^{26}$ K. Pemasiri, M. Montazeri, R. Gass, L. M. Smith, H. E. Jackson, J. Yarrison-Rice, S. Paiman, Q. Gao, H. H. Tan, C. Jagadish, X. Zhang, and J. Zou, Nano Lett. 9, 648 (2009).

${ }^{27}$ T. B. Hoang, A. F. Moses, L. Ahtapodov, H. Zhou, D. L. Dheeraj, A. T. J. van Helvoort, B. -O. Fimland, and H. Weman, Nano Lett. 10, 2927 (2010).

${ }^{28}$ A. Fontcuberta i Morral, C. Colombo, G. Abstreiter, J. Arbiol, and J. R. Morante, Appl. Phys. Lett. 92, 063112 (2008).

${ }^{29}$ D. Spirkoska, C. Colombo, M. Heiss, G. Abstreiter, and A. Fontcuberta i Morral, J. Phys. Condens. Matter 20, 454225 (2008).

${ }^{30}$ C. Colombo, D. Spirkoska, M. Frimmer, G. Abstreiter, and A. Fontcuberta i Morral, Phys. Rev. B 77, 155326 (2008).

${ }^{31}$ J. D. Jackson, Classical Electrodynamics (John Wiley \& Sons Inc., New York, 1999).
${ }^{32}$ J. L. Birman, Phys. Rev. Lett. 2, 157 (1959).

${ }^{33}$ G. L. Bir and G. E. Pikus, Symmetry and strain induced effects in semiconductors (Wiley, New York, 1974).

${ }^{34}$ I. Zardo, S. Conesa-Boj, F. Peiro, J. R. Morante, J. Arbiol, E. Uccelli, G. Abstreiter, and A. Fontcuberta i Morral, Phys. Rev. B 80, 245324 (2009).

${ }^{35}$ Al. L. Efros and A. L. Efros, Sov. Phys. Semicond. 16, 772 (1982).

${ }^{36}$ Al. L. Efros and M. Rosen, Ann. Rev. Mater. Sci. 30, 475 (2000).

${ }^{37}$ Al. L. Efros, T. Birkel, T. Cheiwchanchamnangij, and W. R. L. Lambrecht (unpublished).

${ }^{38}$ J. M. Luttinger and W. Kohn, Phys. Rev. 97, 869 (1955).

${ }^{39}$ J. M. Luttinger, Phys. Rev. 102, 1030 (1956).

${ }^{40}$ P. C. Sereel and K. J. Vahala, Appl. Phys. Lett. 57, 545 (1990).

${ }^{41}$ T. Cheiwchanchamnangij, T. Birkel, W. R. L. Lambrecht, and Al. L. Efros, to be published in Proceedings of the International Conference on Silicon Carbide and Related Materials, 2011, edited by T. P. Chow, R. P. Devaty, and M. Dudley (Trans Tech Publications, Stafa-Zürich, 2012).

${ }^{42}$ D. L. Dheeraj, G. Patriarche, H. Zhou, T. B. Hoang, A. F. Moses, S. Grønsberg, A. T. J. van Helvoort, B.-O. Fimland, and H. Weman, Nano Lett. 8, 4459 (2008).

${ }^{43}$ S. Limpijumnong and W. R. L. Lambrecht, Phys. Rev. B 57, 12017 (1998).

${ }^{44}$ F. R. N. Nabarro, Theory of Crystal Dislocations (Dover Publications, New York, 1987), p. 201.

${ }^{45}$ G. S. Zhdanov, Compt. Rend. Acad. Sci. USSR 48, 43 (1945). 